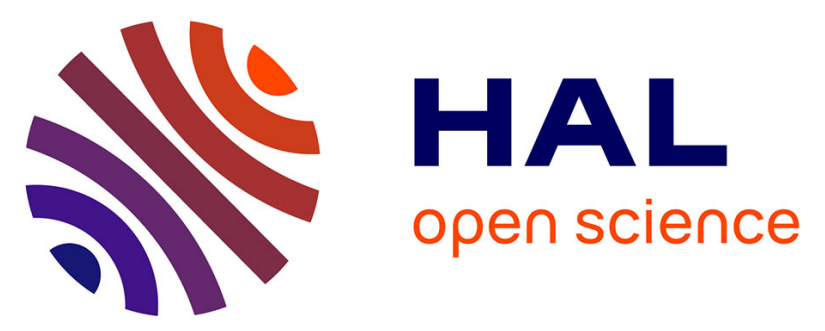

\title{
Capture of instantaneous temperature in oscillating flows: Use of constant-voltage anemometry to correct the thermal lag of cold wires operated by constant-current anemometry
}

Arganthaël Berson, Gaelle Poignand, Philippe Blanc-Benon, Geneviève Comte-Bellot

\section{To cite this version:}

Arganthaël Berson, Gaelle Poignand, Philippe Blanc-Benon, Geneviève Comte-Bellot. Capture of instantaneous temperature in oscillating flows: Use of constant-voltage anemometry to correct the thermal lag of cold wires operated by constant-current anemometry. Review of Scientific Instruments, 2010, 2010, pp.015102. 10.1063/1.3274155 . hal-00566003

\section{HAL Id: hal-00566003 https://hal.science/hal-00566003}

Submitted on 8 Jun 2012

HAL is a multi-disciplinary open access archive for the deposit and dissemination of scientific research documents, whether they are published or not. The documents may come from teaching and research institutions in France or abroad, or from public or private research centers.
L'archive ouverte pluridisciplinaire HAL, est destinée au dépôt et à la diffusion de documents scientifiques de niveau recherche, publiés ou non, émanant des établissements d'enseignement et de recherche français ou étrangers, des laboratoires publics ou privés. 


\title{
Capture of instantaneous temperature in oscillating flows: Use of constant-voltage anemometry to correct the thermal lag of cold wires operated by constant-current anemometry
}

\author{
Arganthaël Berson, ${ }^{1}$ Gaëlle Poignand, ${ }^{2}$ Philippe Blanc-Benon, ${ }^{2}$ and \\ Geneviève Comte-Bellot ${ }^{2}$ \\ ${ }^{1}$ Queen's-RMC Fuel Cell Research Centre, Queen's University, 945 Princess Street, Kingston, \\ Ontario K7L 5L9, Canada \\ ${ }^{2}$ Laboratoire de Mécanique des Fluides et d'Acoustique, UMR CNRS 5509, École Centrale de Lyon, \\ 36 avenue Guy de Collongue, 69134 Ecully Cedex, France
}

(Received 13 July 2009; accepted 21 November 2009; published online 13 January 2010)

\begin{abstract}
A new procedure for the instantaneous correction of the thermal inertia of cold wires operated by a constant-current anemometer is proposed for oscillating flows. The thermal inertia of cold wires depends both on the wire properties and on the instantaneous incident flow velocity. Its correction is challenging in oscillating flows because no relationship between flow velocity and heat transfer around the wire is available near flow reversal. The present correction procedure requires neither calibration data for velocity nor thermophysical or geometrical properties of the wires. The method relies on the splitting of the time lag of cold wires into two factors, which are obtained using a constant-voltage anemometer in the heated mode. The first factor, which is intrinsic to the wire, is deduced from time-constant measurements performed in a low-turbulence flow. The second factor, which depends on the instantaneous flow velocity, is acquired in situ. In oscillating flows, data acquisition can be synchronized with a reference signal so that the same wire is alternatively operated in the cold mode by a constant-current anemometer and in the heated mode by a constant-voltage anemometer. Validation experiments are conducted in an acoustic standing-wave resonator, for which the fluctuating temperature field along the resonator axis is known independently from acoustic pressure measurements, so that comparisons can be made with cold-wire measurements. It is shown that despite the fact that the wire experiences flow reversal, the new procedure recovers accurately the instantaneous temperature of the flow. (C) 2010 American Institute of Physics. [doi:10.1063/1.3274155]
\end{abstract}

\section{INTRODUCTION}

Fine-wire sensors operated at very low overheat ratios act as resistance thermometers. They are usually called "cold wires" and permit the investigation of temperature fluctuations in turbulent flows. For such applications, they are usually preferred to thermocouples because their small size provides a better frequency response. However, they are not without thermal inertia. Moreover, they are usually operated by constant-current anemometers (CCAs), which do not offer an internal hardware compensation. Therefore, CCA data have to be processed numerically a posteriori in order to include a proper correction.

As recalled in Appendix A, the basic first-order differential equation that connects the actual wire resistance $R_{\mathrm{CCA}}(t)$ to the resistance $R_{\mathrm{CCA}}^{*}(t)$, which would be given by an ideal wire without thermal lag, is

$$
\mathcal{M}_{\mathrm{CCA}}(t) \frac{d R_{\mathrm{CCA}}(t)}{d t}+R_{\mathrm{CCA}}(t)=R_{\mathrm{CCA}}^{*}(t),
$$

where $t$ is time and $\mathcal{M}_{\mathrm{CCA}}$ the so-called "time constant" of the cold wire, which, in fact, is time-dependent. In what follows, we will refer to this function as the time lag. Only the resistance $R_{\mathrm{CCA}}^{*}(t)$ of the ideal wire is connected to the instantaneous flow temperature $T_{a}(t)$ by the relation

$$
T_{a}(t)-T_{0}=\frac{R_{\mathrm{CCA}}^{*}(t)-R_{0}}{R_{0} \chi},
$$

where $\chi$ is the temperature coefficient of the electric resistivity of the wire material and $R_{0}$ is the wire resistance at the reference temperature $T_{0}$.

In order to restore $R_{\mathrm{CCA}}^{*}(t)$ from $R_{\mathrm{CCA}}(t)$ using Eq. (1), it is essential to know $\mathcal{M}_{\mathrm{CCA}}(t)$. The $\mathcal{M}_{\mathrm{CCA}}(t)$ of a cold wire of large enough aspect ratio and operated in a constant-current mode is given by [see also Eq. (A7)]

$$
\mathcal{M}_{\mathrm{CCA}}(t)=\frac{m_{w} c_{w}}{\chi R_{0}} \frac{1}{f[U(t)]} .
$$

Clearly, $\mathcal{M}_{\mathrm{CCA}}(t)$ depends on the wire properties via the factor $m_{w} c_{w} / \chi R_{0}$, but also on the flow velocity via the factor $1 / f[U(t)]$. Symbols are defined in the Nomenclature section.

Let us now examine how $\mathcal{M}_{\mathrm{CCA}}$ has been taken into account so far.

- (i) The simplest approach is to assume $\mathcal{M}_{\mathrm{CCA}} \simeq 0$. This is possible for small turbulence levels, low flow velocities, and very thin wires. Indeed, for the wire thermal inertia to be negligible, the cutoff frequency $f_{c}$ of the wire should be much greater than the greatest frequency of interest, namely, the Kolmogorov frequency $f_{K}$ that characterizes 
the smallest turbulent structures to be resolved. At low fluid velocity, the Kolmogorov frequency is low and thin wires have a high cutoff frequency, so that these conditions ensure $f_{c} \gg f_{K}$. For example, Yeh and Van Atta ${ }^{1}$ chose a wire of diameter $0.25 \mu \mathrm{m}$ to investigate temperature spectra and higher moments behind a heated grid, at a mean flow velocity of $4 \mathrm{~m} \cdot \mathrm{s}^{-1}$. In their case, $f_{K}=1 \mathrm{kHz}$ and $f_{c}$ $=5 \mathrm{kHz}$. Mydlarski and Warhaft ${ }^{2}$ selected a $0.63 \mu \mathrm{m}$ wire for the study of temperature fluctuations behind a heated active grid at $\bar{U} \leq 10 \mathrm{~m} . \mathrm{s}^{-1}$.

- (ii) Another approach consists in using the time-averaged value of $\mathcal{M}_{\mathrm{CCA}}(t)$, denoted $\overline{\mathcal{M}}_{\mathrm{CCA}}$. This assumes that velocity fluctuations are of small amplitude relative to $\bar{U}$ so that $\mathcal{M}_{\mathrm{CCA}}(t) \simeq \overline{\mathcal{M}}_{\mathrm{CCA}}$ around the mean operating point. This approach requires the measurement of $\overline{\mathcal{M}}_{\mathrm{CCA}}$ at every location of interest in the flow. Various methods have been used to obtain $\overline{\mathcal{M}}_{\mathrm{CCA}}$, e.g., a square-wave current injection, a chopped laser beam focused on the wire, a short pulse voltage applied in an upstream wire, and an oscillation of the wire between a hot zone and a cold one. Details are indicated by Antonia et al., ${ }^{3}$ Lemay and Benaïssa, ${ }^{4}$ Lemay et al., ${ }^{5}$ and LaRue et al. ${ }^{6}$ Note that for the first two methods, great care should be taken not to heat the wire excessively. ${ }^{5,7}$

- (iii) Bremhorst and Graham, ${ }^{8}$ Graham and Bremhorst, ${ }^{9}$ and Vukoslavčević and Wallace ${ }^{10}$ attempted to obtain the instantaneous values of $\mathcal{M}_{\mathrm{CCA}}(t)$ for flows having a well defined direction of development. An additional wire is placed close to the cold wire. This additional wire operates in the heated mode and its characteristics do not have to be necessarily the same as those of the cold wire. The hot wire is operated by a constant-temperature anemometer and gives $U(t)$ by proper calibration. Then $f[U(t)]$ is obtained from a heat transfer-velocity relationship. Such a relationship exists only when forced convection prevails and is explicited by a Nusselt-Reynolds diagram or by King's law (or similar).

- (iv) Acoustic standing waves, oscillating or pulsed flows such as in synthetic jets or blood vessels, introduce an additional difficulty compared to case (iii) because the wire now experiences flow reversal. Near flow reversal, no heat transfer-velocity relationship is available so that $\mathcal{M}_{\mathrm{CCA}}(t)$ cannot be calculated from hot-wire velocity measurements. Pedley ${ }^{11}$ raised this issue already in 1975. Elger and Adams ${ }^{12}$ investigated the spatially averaged instantaneous Nusselt number of a wire exposed to an oscillating flow up to $70 \mathrm{~Hz}$ but their results are difficult to generalize. Most experimental researchers went back to the simplest approach $\mathcal{M}_{\mathrm{CCA}} \simeq 0$, e.g., Huelsz and Ramos ${ }^{13}$ in an acoustic oscillatory boundary layer at low frequency -130 $\mathrm{Hz}$, or Berson ${ }^{14}$ and Blanc-Benon and Berson ${ }^{15}$ in a thermoacoustic refrigerator. These authors indicated that the measured amplitudes of temperature fluctuations were largely underestimated.

In the present work, we propose a procedure for the measurements of temperature fluctuations in oscillating flow-case (iv) above-for which no satisfactory method for temperature measurements exists so far. As an example, the characterization of temperature fluctuations in oscillating flows is crucial in thermoacoustic systems, including pulsetube coolers and Stirling engines, in order to optimize the design and performances of such devices. The key feature of the new procedure lies in the accurate measurement of the instantaneous time lag $\mathcal{M}_{\mathrm{CCA}}(t)$ of the cold wire. This is achieved by using a constant-voltage anemometer (CVA) operating the same wire in the heated mode.

Section II shows how the two factors $m_{w} c_{w} / \chi R_{0}$ and $1 / f[U(t)]$ making up $\mathcal{M}_{\mathrm{CCA}}(t)$ can be obtained for the cold wire, without requiring the knowledge of either the wire properties or a heat transfer-velocity relationship. The full experimental procedure for the instantaneous correction of temperature signals is described in Sec. III. Validations are presented in Sec. IV for the temperature field in an acoustic standing-wave resonator. In the resonator, temperature oscillations can be calculated from the pressure field so that they provide a reference for comparison with cold-wire measurements. With the new procedure, measurements are not limited to the fundamental frequency. Higher harmonics of temperature fluctuations that arise from nonlinear acoustic effects can also be investigated. Conclusions are given in Sec. V. The governing equations for a cold wire operated by a CCA and a hot wire operated by a CVA are summarized in Appendix A and Appendix B, respectively.

\section{DETERMINATION OF $\mathcal{M}_{\text {CCA }}(t)$ USING CONSTANT- VOLTAGE ANEMOMETRY}

In this section, the wire is operated in the heated mode by a CVA. The main characteristics and the governing equations of a CVA are given in Appendix B. The two factors $m_{w} c_{w} / \chi R_{0}$ and $1 / f[U(t)]$ that compose $\mathcal{M}_{\mathrm{CCA}}(t)$, according to Eq. (3), are obtained in the following way.

\section{A. Determination of $m_{w} c_{w} / \chi R_{0}$}

The coefficient $m_{w} c_{w} / \chi R_{0}$ is given by Eq. (B8)

$$
\frac{m_{w} c_{w}}{\chi R_{0}}=\bar{M}_{\mathrm{CVA}} V_{w}^{2} \frac{1}{\left(\bar{R}_{\mathrm{CVA}}+r_{L}\right)^{2}} \frac{1+2 \bar{a}_{w}}{\bar{a}_{w}} \frac{1}{L M} .
$$

Measurements are made in the potential core of a subsonic jet where $T_{a}$ is constant $T_{a}=T_{0}$. Since the turbulence level is very low, $\bar{R}_{\mathrm{CVA}}=R_{\mathrm{CVA}}^{*}$ so that the differential Eq. (B4) does not have to be solved and $\bar{R}_{\mathrm{CVA}}$ is straightforwardly deduced from $\bar{E}_{\mathrm{CVA}}$ using Eq. (B1), without the timederivative term. The time constant $\bar{M}_{\mathrm{CVA}}$ is measured using the square-wave test circuit included in the CVA unit. ${ }^{16,17}$ The factor $L M$, which takes into account the resistance of the lead, is evaluated from Eq. (B7).

Note that the determination of $m_{w} c_{w} / \chi R_{0}$ does not require the knowledge of either the geometric properties of the wire or the physical and electrical properties of the material constituting the wire. This is all the most important regarding the wire diameter. It appears with a fourth power in $m_{w} c_{w} / \chi R_{0}$ since $m_{w}=\pi \rho_{w} l_{w} d_{w}^{2} / 4$ and $R_{0}=4 \rho_{e} l_{w} / \pi d_{w}^{2}$, and its actual value, which can be measured by an electron microscope, for example, always differs from the specifications announced by manufacturers. 
The benefits of this method for the determination of $m_{w} c_{w} / \chi R_{0}$ were recently illustrated by Berson et al. ${ }^{18}$ in order to eliminate all nonlinearities of a CVA at large velocity changes.

\section{B. Determination of $1 / f[U(t)]$}

The determination of the factor $1 / f[U(t)]$ makes use of Eq. (B9) rewritten as

$$
\frac{1}{f[U(t)]}=\frac{\left(R_{\mathrm{CVA}}^{*}+r_{L}\right)^{2}}{V_{w}^{2}} \frac{R_{\mathrm{CVA}}^{*}-R_{a}}{R_{\mathrm{CVA}}^{*}} .
$$

All quantities are acquired in situ in the flow under investigation. This only requires $V_{w}$ to be chosen and $E_{\mathrm{CVA}}(t)$ to be recorded. During post-processing, $R_{\mathrm{CVA}}(t)$ is obtained from $E_{\mathrm{CVA}}(t)$ using Eq. (B1), then $R_{\mathrm{CVA}}(t)$ gives $R_{\mathrm{CVA}}^{*}(t)$ using Eq. (B4), the factor $m_{w} c_{w} / \chi R_{0}$ being known from Sec. II A. $R_{a}$ is taken at the previous time step as the sampling frequency can be chosen much larger than the highest frequency to be resolved in the investigated field.

\section{OBTAINING THE FLOW TEMPERATURE $T_{a}(t)$}

As explained in Sec. I, the basic equation Eq. (1) is solved in order to obtain the resistance $R_{\mathrm{CCA}}^{*}$ of the ideal cold wire, from which the incident flow temperature $T_{a}(t)$ is obtained using Eq. (2) and the value of $\chi$. This approach requires two measurements: (i) the output voltage $E_{\mathrm{CCA}}(t)$ of the CCA operating in the cold mode, which is used to obtain the uncorrected resistance $R_{\mathrm{CCA}}(t)$ of the cold wire, as explained in Appendix A, Eq. (A9)

$$
R_{\mathrm{CCA}}(t)=\frac{E_{\mathrm{CCA}}(t)+G E_{\mathrm{offset}}}{100 G I_{\mathrm{CCA}}}-r_{L} ;
$$

and (ii) the output voltage $E_{\mathrm{CVA}}(t)$ of the CVA operating in the heated mode, which leads to the instantaneous time lag $\mathcal{M}_{\mathrm{CCA}}(t)$ of the cold wire, as explained in Sec. II.

Assuming that the flow is periodic, the procedure requires only one wire. Data acquisition is synchronized with a reference signal that is characteristic of the periodic flow, e.g., pressure measurements. The same wire is operated alternately as a cold wire by a CCA and as a hot wire by a CVA so as to produce the two output signals that are needed, $E_{\mathrm{CCA}}(t)$ and $E_{\mathrm{CVA}}(t)$.

The whole experimental procedure can be summarized as follows:

- Prior to the tests:

1. Measure $R_{0}$ and $r_{L}$, at a reference temperature $T_{0}$;

2. Measure $\chi$ in a thermostat;

3. Choose $I_{\mathrm{CCA}}$ to operate the cold wire in a CCA mode, note $\mathrm{G}$ and $E_{\text {offset; }}$;

4. Choose $V_{w}$ and $T_{C}$ to operate the wire now heated in a CVA mode;

5. Measure the time constant $\bar{M}_{\mathrm{CVA}}$ of the heated wire in a constant-velocity flow, using the square-wave test unit of the CVA;

6. Deduce $m_{w} c_{w} / \chi R_{0}$ using Eq. (4).

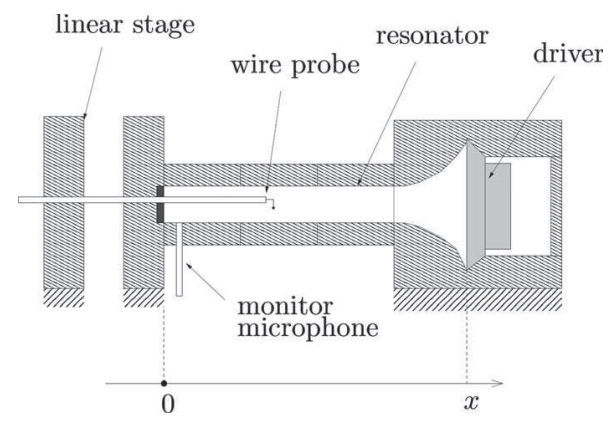

FIG. 1. Schematic drawing of the experimental setup.

- During the tests:

1. Acquire $E_{\mathrm{CCA}}(t)$ in situ for the cold wire, the CCA being set at the same $I_{\mathrm{CCA}}$ value as above;

2. Acquire $E_{\mathrm{CVA}}(t)$ in situ for the hot wire, the CVA being set at the same $V_{w}$ and $T_{C}$ values as above. The flow being periodic, $E_{\mathrm{CCA}}(t)$ and $E_{\mathrm{CVA}}(t)$ are acquired successively with the same wire.

- During postprocessing:

1. Calculate $1 / f[U(t)]$ using this order: Eq. (B1) to obtain $R_{\mathrm{CVA}}$, Eq. (B4) to obtain $R_{\mathrm{CVA}}^{*}$, and Eq. (5) to obtain $1 / f[U(t)]$. At each time step the value of $R_{a}$ is taken at the previous time increment.

2. Calculate the instantaneous time lag $\mathcal{M}_{\mathrm{CCA}}(t)$, according to Eq. (3);

3. Solve Eq. (1) to obtain $R_{\mathrm{CCA}}^{*}$ from $R_{\mathrm{CCA}}$;

4. Deduce the instantaneous temperature $T_{a}$ from $R_{\mathrm{CCA}}^{*}$ using the factor $\chi$ and Eq. (2).

Note that the only tuning of the anemometers consists in choosing the values of $I_{\mathrm{CCA}}$ for the CCA, and $V_{w}$ and $T_{C}$ for the CVA. Moreover, all the signals are treated during postprocessing so that the time spent using the experimental facilities is reduced.

\section{VALIDATION: TEMPERATURE FLUCTUATIONS IN AN ACOUSTIC STANDING WAVE}

\section{A. Experimental setup}

In order to validate the present procedure for the dynamic correction of the thermal inertia of a cold wire, measurements of temperature fluctuations are performed inside an acoustic resonator. In an acoustic standing wave, a fluid experiences simultaneously pressure, temperature, and velocity oscillations, which makes it suitable for our demonstration. ${ }^{14,15}$

A schematic drawing of the experimental setup is given in Fig. 1. The acoustic standing wave is generated in a closed resonator of total length $18 \mathrm{~cm}$. The resonator consists of a 15-cm-long Plexiglas tube, with a cylindrical cross-section of diameter $3 \mathrm{~cm}$. An exponential horn connects the tube to the acoustic driver. The tube is filled with air at atmospheric pressure and at ambient temperature $(296 \mathrm{~K})$. The driver is an electrodynamic loudspeaker (GELEC EDM8760F) generating a monotonic sound at the resonance frequency of the 
resonator $f_{\text {osc }}=464 \mathrm{~Hz}$ (quarter-wavelength). The acoustic field inside the resonator is monitored by a fixed 1/4 in. Bruel \& Kjaer microphone flush mounted $0.7 \mathrm{~cm}$ away from the closed end of the resonator. The acoustic pressure level at this location is set to three different values, $P_{\mathrm{ac}}=1000,2000$, and $3000 \mathrm{~Pa}$, or 151,157 , and 160 lindB, respectively, (reference pressure $2 \times 10^{-5} \mathrm{~Pa}$ ).

At such high acoustic pressure levels, higher harmonics are generated inside the resonator, ${ }^{14}$ and especially a second harmonic at the frequency $2 f_{\text {osc }}=928 \mathrm{~Hz}$. The acoustic pressure distribution along the tube is approximated by

$$
\begin{aligned}
P_{\mathrm{ac}}^{\prime}(x, t)= & P_{\mathrm{ac}}\left[\cos (k x) \sin \left(\omega t+\Phi_{1}\right)\right. \\
& \left.+\epsilon \cos (2 k x) \sin \left(2 \omega t+\Phi_{2}\right)\right],
\end{aligned}
$$

where $k=2 \pi f_{\text {osc }} / c_{0}$ is the acoustic wave number, with $c_{0}$ the speed of sound in air. In order to obtain a precise description of the acoustic pressure distribution along the tube, a direct measurement of $P_{\mathrm{ac}}^{\prime}$ is performed using a $1 / 4$ in. Bruel \& Kjaer microphone that is displaced along the straight part of the tube. The relative amplitude of the second harmonic is found to be $\epsilon=0.02,0.03,0.05$, respectively, for $P_{\mathrm{ac}}=1000$, 2000 , and $3000 \mathrm{~Pa}$. The reference for the measurements of phases $\Phi_{1}$ and $\Phi_{2}$ is taken as the fundamental frequency of the monitor microphone.

The velocity fluctuations in the tube are related to the amplitude of the acoustic pressure by

$$
\begin{aligned}
U_{\mathrm{ac}}^{\prime}(x, t)= & \frac{P_{\mathrm{ac}}}{\rho_{m} c_{0}}\left[\sin (k x) \cos \left(\omega t+\Phi_{1}\right)\right. \\
& \left.+\epsilon \sin (2 k x) \cos \left(2 \omega t+\Phi_{2}\right)\right],
\end{aligned}
$$

where $\rho_{m}$ is the mean density of the fluid. Velocity fluctuations, which are $\pi / 2$ out-of-phase with pressure fluctuations, present large amplitude. In the present experiments, the maximal incident velocity on the wire varies over the ranges \pm 2.5 , \pm 4.6 , and $\pm 6.9 \mathrm{~m} . \mathrm{s}^{-1}$, respectively, for the three pressure levels considered.

The temperature fluctuations relevant to the present investigation are in phase with pressure fluctuations since they obey an adiabatic process. Their spatial distribution along the tube is therefore given by

$$
T_{\mathrm{ac}}^{\prime}(x, t)=\frac{\gamma-1}{\gamma} \frac{T_{m}}{P_{m}} P_{\mathrm{ac}}^{\prime}(x, t),
$$

where $\gamma$ is the specific heat ratio of the fluid, $T_{m}$ is the mean ambient temperature, $T_{m}=\bar{T}_{a} \simeq T_{0} . P_{m}$ is the mean ambient pressure-in our case the atmospheric pressure. In the following, the profiles of temperature amplitudes and phases calculated from pressure measurements serve as references for comparison with cold-wire measurements.

\section{B. Wire and anemometers}

The wire, made of pure tungsten, is mounted on an L-shaped probe (Dantec 55P04). Its diameter is $d_{w} \approx 3 \mu \mathrm{m}$ measured by a scanning electron microscope and its length is $l_{w}=3 \mathrm{~mm}$. The aspect ratio $\left(l_{w} / d_{w} \approx 1000\right)$ is considered large enough for end-conduction effects to be neglected. The probe is inserted in the resonator through a rubber membrane

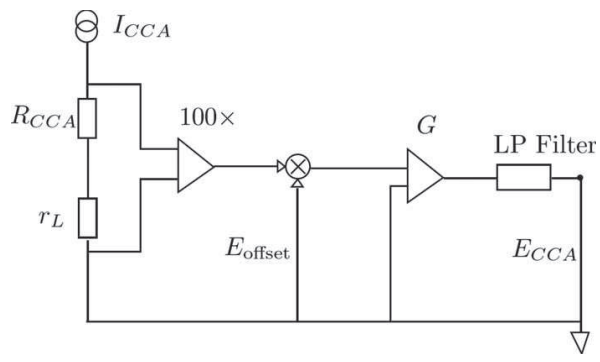

FIG. 2. Schematic drawing of the Dantec 90C20 temperature module.

embedded in the closed end wall in order to ensure a proper sealing. The wire is displaced along the $x$-axis of the resonator using an accurate linear stage.

The wire resistance at room temperature $\left(T_{0}=296 \mathrm{~K}\right)$ is $R_{0}=29.1 \Omega$ and the temperature coefficient of resistivity has been measured to be $\chi=0.0035 \mathrm{~K}^{-1}$. The resistance of the connection lead is $r_{L}=0.47 \Omega$ and $L M \approx 1$.

Cold-wire measurements are performed using the temperature module of a Dantec Streamline 90C20 anemometer (Fig. 2). Three current intensities are used $I_{\mathrm{CCA}}=0.1,0.2$ and $0.5 \mathrm{~mA}$. The offset voltage $E_{\text {offset }}$ is adjusted and measured for each current. The dc gain is set to $G=400$ and the lowpass filter to $3 \mathrm{kHz}$ (position off).

The wire, when heated, is connected to a CVA, model VC01 from Tao Systems (Fig. 3). The voltage across the wire and its lead is set to $V_{w}=800 \mathrm{mV}$ and the hardware compensation is $T_{C}=100 \mu \mathrm{s}$.

In the present experiment, it is possible to use the same wire because acoustic fluctuations are periodic. Hot-wire and cold-wire measurements are synchronized with the pressure given by the monitor microphone. For each acoustic pressure level and different wire locations along the tube, two successive runs are performed, one in the constant-current coldwire mode, the other in the constant-voltage hot-wire mode. Data are recorded at a sampling frequency of $f_{s}=25.6 \mathrm{kHz}$. Electronic noise and low-frequency fluctuations are removed by reconstructing the Fourier series of the signals for the first 15 harmonics of the frequency of oscillation.

Before presenting the results that deal with the temperature field inside the resonator, let us indicate the value of the factor $m_{w} c_{w} / \chi R_{0}$ of the wire. Table I shows values obtained for different velocities and different overheat ratios. All the tests show that a constant value $m_{w} c_{w} / \chi R_{0} \simeq 1.04$ $\times 10^{-6}$ s. $\mathrm{A}^{2}$ is obtained.

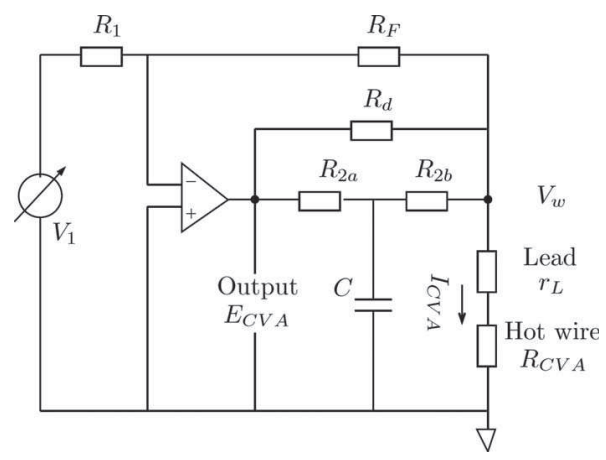

FIG. 3. Schematic drawing of a CVA. 
TABLE I. Values of $m_{w} c_{w} / \chi R_{0}$ measured in the potential core of a subsonic jet for different velocities and overheat ratios.

\begin{tabular}{cccccccc}
\hline \hline $\begin{array}{c}U \\
\left(\mathrm{~m} . \mathrm{s}^{-1}\right)\end{array}$ & $\begin{array}{c}V_{w} \\
(\mathrm{mV})\end{array}$ & $\begin{array}{c}\bar{E}_{\mathrm{CVA}} \\
(\mathrm{V})\end{array}$ & $\begin{array}{c}\bar{M}_{\mathrm{CVA}} \\
(\mu \mathrm{s})\end{array}$ & $\begin{array}{c}\bar{R}_{\mathrm{CVA}} \\
(\Omega)\end{array}$ & $\bar{a}_{w}$ & $L M$ & $\begin{array}{c}m_{w} c_{w} / \chi R_{0} \\
\left(\mu \mathrm{s} . \mathrm{A}^{2}\right)\end{array}$ \\
\hline \multirow{4}{*}{2.2} & 700 & 2.969 & 404 & 32.9 & 0.25 & 1.00 & 1.06 \\
& 800 & 3.283 & 376 & 35.6 & 0.31 & 1.01 & 1.02 \\
& 900 & 3.572 & 368 & 36.3 & 0.37 & 1.01 & 1.02 \\
4 & 700 & 3.016 & 348 & 32.2 & 0.22 & 1.00 & 1.04 \\
& 800 & 3.342 & 336 & 33.7 & 0.28 & 1.00 & 1.03 \\
& 900 & 3.647 & 324 & 35.2 & 0.33 & 1.01 & 1.03 \\
6 & 700 & 3.050 & 324 & 31.7 & 0.20 & 1.00 & 1.07 \\
& 800 & 3.386 & 308 & 33.1 & 0.25 & 1.00 & 1.04 \\
& 900 & 3.696 & 298 & 34.5 & 0.31 & 1.01 & 1.03 \\
\hline \hline
\end{tabular}

\section{Results}

The contribution brought to $T_{\mathrm{ac}}^{\prime}$ at the fundamental frequency $f_{\text {osc }}$ is denoted by $T_{1}$ for its amplitude and by $\phi_{1}$ for its phase relative to $\Phi_{1}$. Similarly, the contribution of the second harmonic, at $2 f_{\text {osc }}$, is denoted by $T_{2}$ for its amplitude and by $\phi_{2}$ for its phase relative to $\Phi_{2}$. Therefore, it is expected that correctly compensated CCA data will agree with Eq. (8), i.e.,

$$
\begin{aligned}
& T_{1}=\frac{\gamma-1}{\gamma} \frac{T_{m}}{P_{m}} P_{\mathrm{ac}} \cos (k x) \quad \text { and } \quad \phi_{1}=0, \\
& T_{2}=\epsilon \frac{\gamma-1}{\gamma} \frac{T_{m}}{P_{m}} P_{\mathrm{ac}} \cos (2 k x) \text { and } \phi_{2}=0 .
\end{aligned}
$$

Figure 4 shows the distributions of $T_{1}$ along the axis of the resonator. Data presented on the left-hand side of Fig. 4 are not corrected (NC) whereas data on the right-hand side are corrected (C). The use of the same ordinate scale on both
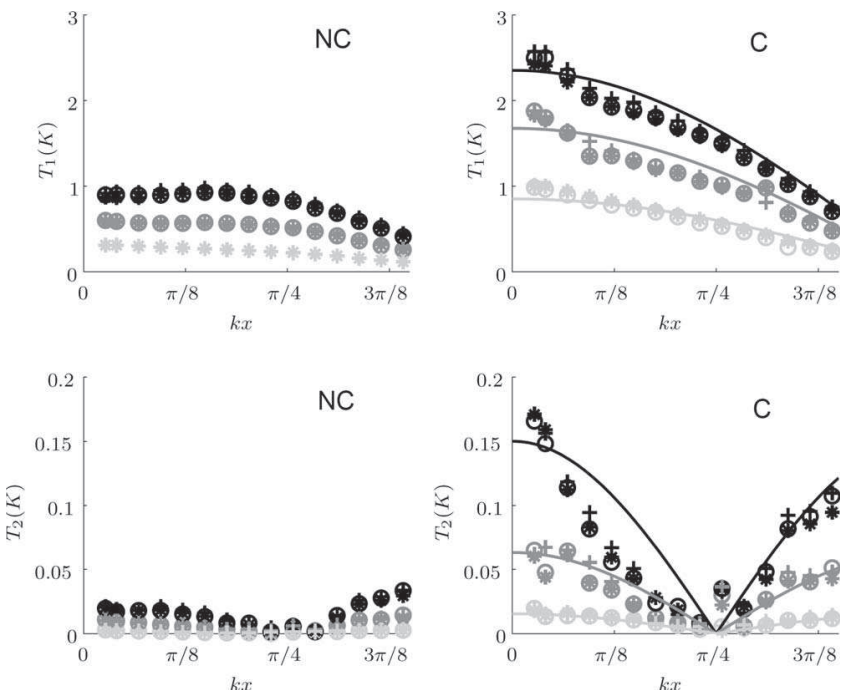

FIG. 4. Amplitudes of temperature fluctuations $T_{1}$ at the fundamental frequency $f_{\text {osc }}$ and $T_{2}$ at the second harmonic $2 f_{\text {osc }}$ with correction (C) and without correction (NC) measured along the axis of the acoustic resonator for $P_{\mathrm{ac}}=1000$ (light gray), 2000 (dark gray), and $3000 \mathrm{~Pa}$ (black) and for different currents $\bigcirc: 0.1 \mathrm{~mA}, \square: 0.2 \mathrm{~mA}$, and +: $0.5 \mathrm{~mA}$. Full lines correspond to Eq. (9). plots highlights the fact that uncorrected data largely underestimate - by a factor of 3 approximately-temperature fluctuations. The dynamic correction dramatically improves the agreement with the theoretical curves given by Eq. (9). The distribution of $T_{1}$, with a maximal amplitude at the close end of the resonator, at $k x=0$, and zero temperature fluctuations close to the loudspeaker, at $k x \simeq \pi / 2$, is well recovered.

The axial distributions of $T_{2}, \mathrm{NC}$ and $\mathrm{C}$, are also indicated on Fig. 4 with the same ordinate scale for both. Note the very small amplitude of uncorrected temperature fluctuations, not more than $0.02 \mathrm{~K}$ approximately, i.e., a factor of about 8 between corrected and uncorrected values. The restored values are in close agreement with the theoretical curves. $T_{2}$ is maximum near both the closed end of the resonator and the loudspeaker. The node at $k x=\pi / 4$ is also well observed.

Results for the phases $\phi_{1}$ and $\phi_{2}$ are reported in Fig. 5. Uncorrected values are approximately $-\pi / 2$ out-of-phase with theoretical data, which is the high-frequency limit for a
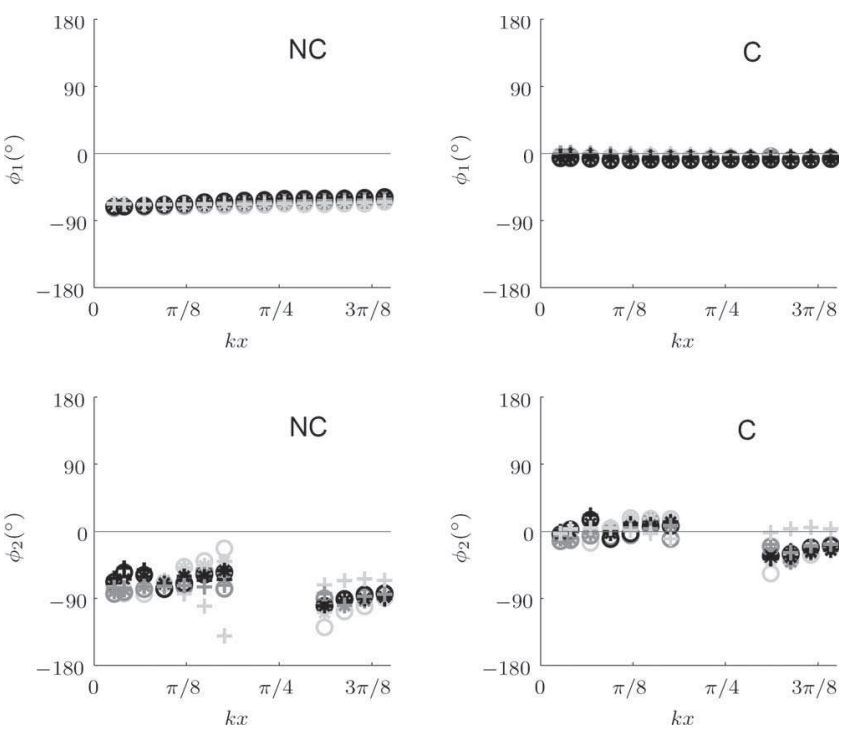

FIG. 5. Phases of temperature fluctuations $\phi_{1}$ at the fundamental frequency $f_{\text {osc }}$ and $\phi_{2}$ at the second harmonic $2 f_{\text {osc }}$ with correction $(\mathrm{C})$ and without correction (NC) measured along the axis of the acoustic resonator for $P_{\mathrm{ac}}$ $=1000$ (light gray), 2000 (dark gray), and $3000 \mathrm{~Pa}$ (black) and for different currents $\bigcirc: 0.1 \mathrm{~mA}, \square: 0.2 \mathrm{~mA}$, and +: $0.5 \mathrm{~mA}$. 


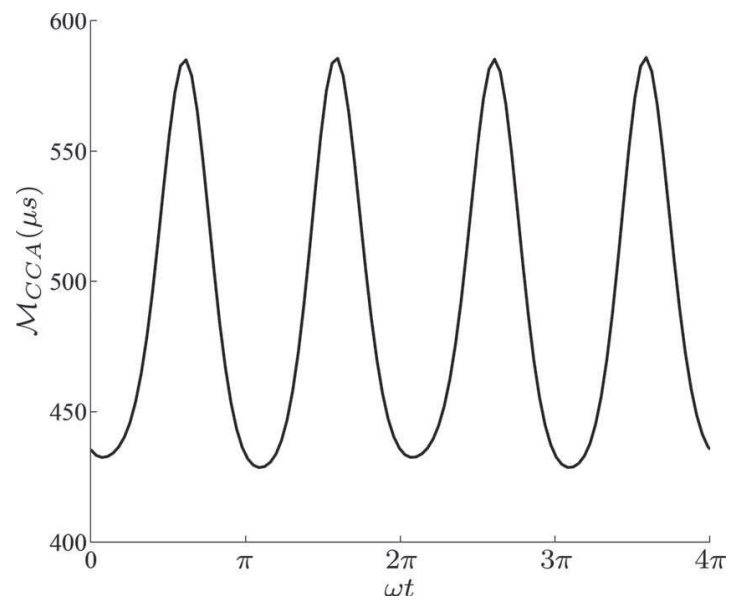

FIG. 6. Temporal evolution of the time lag $\mathcal{M}_{\mathrm{CCA}}(t)$ of the wire for $P_{\mathrm{ac}}$ $=3000 \mathrm{~Pa}$ and $k x=3 \pi / 8$.

wire operated in the constant-current mode. The corrected phases, on the right-hand side, are close to zero, as expected. In the vicinity of the node of $T_{2}$, the raw CCA values are very small and preclude any accurate phase detection. That part, located around $k x=\pi / 4$, is therefore omitted on the diagram.

Figure 6 shows an example of the temporal evolution of the time lag $\mathcal{M}_{\mathrm{CCA}}(t)$. At the chosen location, $k x=3 \pi / 8$, large-amplitude velocity oscillations occur, approximately $\pm 6 \mathrm{~ms}^{-1}$, which explains the large fluctuations of $\mathcal{M}_{\mathrm{CCA}}(t)$, between $450 \mu$ s at maximum velocity and $600 \mu$ s near flow reversal. Note that $\mathcal{M}_{\mathrm{CCA}}(t)$ oscillates at twice the acoustic frequency since the wire does not take into account the direction of the incident velocity.

\section{CONCLUSION}

A new procedure based on the joint use of a CCA operating a cold wire and of a CVA operating the same wire in a heated mode is proposed for the measurement of temperature fluctuations in oscillating flows. The CVA measures the instantaneous time lag of the cold wire that is necessary for the correction of temperature signals acquired with the CCA. Temperature fluctuations up to around $1000 \mathrm{~Hz}$ have been measured with a robust wire whose diameter is approximately $3 \mu \mathrm{m}$. The idea that only thin wires, around $1 \mu \mathrm{m}$ or less, could perform measurements at such frequency is shown to be obsolete. Extension of the suggested procedure to other types of unsteady flows such as jet flows or boundary layers is presently under investigation.

\section{ACKNOWLEDGMENTS}

The authors are grateful to Dr. Siva Mangalam from Tao Systems Inc. who permitted the use of a CVA prototype, and to Emmanuel Jondeau for his help with the fabrication of the probes. This work is supported by the French National Research Agency ANR (Project MicroThermAc Grant No. NT051_42101).

\section{Nomenclature}

$c_{w}=$ specific heat of the wire material

$d_{w}=$ wire diameter

$E=$ output voltage of an anemometer for a real wire

$E^{*}=$ output voltage of an anemometer for an ideal wire

$E_{\text {offset }}=$ dc offset voltage in a CCA

$f_{\text {osc }}=$ fundamental frequency of acoustic oscillations

$f(U)=$ a function of $U$ in a heat-transfer law

$G=$ constant dc gain in a CCA

$I=$ current intensity through the wire

$l_{w}=$ wire length

$L M=$ correction term to take into account the lead

$m_{w}=$ mass of the wire

$M=$ time constant at overheat $a_{w}$

$\mathcal{M}=$ limit of $M$ when $a_{w} \rightarrow 0$

$P_{\mathrm{ac}}=$ amplitude of the acoustic pressure in a resonant tube

$P_{\mathrm{ac}}^{\prime}=$ pressure fluctuations in the acoustic resonator

$R=$ resistance of a real wire

$R_{a}=$ wire resistance at temperature $T_{a}$

$R_{0}=$ wire resistance at temperature $T_{0}$

$R_{1}, R_{2}, R_{2 a}, R_{2 b}, R_{F}=$ resistances in the CVA circuit

$R^{*}=$ resistance of an ideal wire

$r_{L}=$ lead resistance

$T_{C}=$ hardware time lag correction in the CVA circuit

$T_{a}=$ incident flow temperature

$T_{\mathrm{ac}}^{\prime}=$ temperature fluctuations in the acoustic resonator

$T_{0}=$ reference temperature

$T_{1}=$ contribution to $T_{\mathrm{ac}}^{\prime}$ at frequency $f_{\mathrm{osc}}$

$T_{2}=$ contribution to $T_{\mathrm{ac}}^{\prime}$ at frequency $2 f_{\mathrm{osc}}$

$t=$ time

$U=$ velocity component normal to the wire

$U_{\mathrm{ac}}^{\prime}=$ velocity fluctuations in the acoustic resonator

$V_{w}=$ voltage across the wire, kept constant in CVA

\section{Greek symbols}

$\chi=$ temperature coefficient of resistivity for the wire material

$\Phi_{1}=$ phase of $T_{\mathrm{ac}}^{\prime}$ relative to the reference microphone at frequency $f_{\text {osc }}$

$\Phi_{2}=$ phase of $T_{\mathrm{ac}}^{\prime}$ relative to the reference microphone at frequency $2 f_{\text {osc }}$

$\phi_{1}=$ phase for $T_{1}$ relative to $\Phi_{1}$ at frequency $f_{\text {osc }}$

$\phi_{2}=$ phase for $T_{2}$ relative to $\Phi_{2}$ at frequency $2 f_{\text {osc }}$

$\rho_{e}=$ electric resistivity of the wire material

$\rho_{w}=$ density of the wire material

$\omega=$ angular frequency 


\section{Subscripts}

$\mathrm{CCA}=$ constant-current anemometer

$\mathrm{CVA}=$ constant-voltage anemometer

\section{APPENDIX A: BASIC CCA EQUATIONS FOR A COLD WIRE}

In a constant-current mode, the heat balance of an ideal wire, i.e., with no thermal lag, is simply

$$
0=R_{\mathrm{CCA}}^{\star} I_{\mathrm{CCA}}^{2}-\left(R_{\mathrm{CCA}}^{\star}-R_{a}\right) f[U(t)],
$$

and the heat balance of a real wire with thermal lag is

$$
\frac{m_{w} c_{w}}{\chi R_{0}} \frac{d R_{\mathrm{CCA}}}{d t}=R_{\mathrm{CCA}} I_{\mathrm{CCA}}^{2}-\left(R_{\mathrm{CCA}}-R_{a}\right) f[U(t)],
$$

where $I_{\mathrm{CCA}}$ is the constant current intensity in either wire (at this point not necessarily small), $R_{\mathrm{CCA}}^{\star}(t)$ is the instantaneous resistance of the ideal wire, $R_{\mathrm{CCA}}(t)$ is the instantaneous resistance of the real wire, and $R_{a}$ is the resistance of either wire at the incident flow temperature $T_{a}$. For the other notations, see the Nomenclature section. On the right-hand side of Eqs. (A1) and (A2), the first term is the Joule heating of the wire and the second term is the heat loss to the flow. The thermal energy stored in the real wire appears in the lefthand side of Eq. (A2). Heat losses at the ends of the wires are neglected. This assumes a large value of the wire aspect ratio $l_{w} / d_{w}$ as indicated by Bremhorst and Gilmore ${ }^{7}$ or LaRue et al. ${ }^{6}$ In the present investigation this condition is satisfied since $l_{w} / d_{w} \simeq 1000$.

Equations (A1) and (A2) can be rewritten as

$$
\begin{aligned}
& 0=\left(R_{\mathrm{CCA}}^{\star}-R_{a}\right)\left\{I_{\mathrm{CCA}}^{2}-f[U(t)]\right\}+R_{a} I_{\mathrm{CCA}}^{2}, \\
& \frac{m_{w} c_{w}}{\chi R_{0}} \frac{d R_{\mathrm{CCA}}}{d t}=\left(R_{\mathrm{CCA}}-R_{a}\right)\left\{I_{\mathrm{CCA}}^{2}-f[U(t)]\right\}+R_{a} I_{\mathrm{CCA}}^{2} .
\end{aligned}
$$

Subtracting Eqs. (A3) and (A4) gives

$$
\frac{m_{w} c_{w}}{\chi R_{0}} \frac{1}{\left\{f[U(t)]-I_{\mathrm{CCA}}^{2}\right\}} \frac{d R_{\mathrm{CCA}}}{d t}+R_{\mathrm{CCA}}=R_{\mathrm{CCA}}^{*} .
$$

The factor in front of $d R_{\mathrm{CCA}} / d t$ is the time lag of the wire operated in the constant-current mode. It is usually noted $M_{\mathrm{CCA}}(t)$ and Eq. (A5) gives

$$
M_{\mathrm{CCA}}(t)=\frac{m_{w} c_{w}}{\chi R_{0}} \frac{1}{\left\{f[U(t)]-I_{\mathrm{CCA}}^{2}\right\}} .
$$

For a cold wire, the current intensity $I_{\mathrm{CCA}}$ is very small and, in this limit, $M_{\mathrm{CCA}}(t)$ becomes $\mathcal{M}_{\mathrm{CCA}}(t)$, expressed as

$$
\mathcal{M}_{\mathrm{CCA}}(t)=\frac{m_{w} c_{w}}{\chi R_{0}} \frac{1}{f[U(t)]} .
$$

$\mathcal{M}_{\mathrm{CCA}}(t)$ is clearly made of two factors: $m_{w} c_{w} / \chi R_{0}$, which is intrinsic to the wire, and $1 / f[U(t)]$, which depends on the instantaneous velocity of the incident flow. Equation (A5) thus becomes

$$
\mathcal{M}_{\mathrm{CCA}}(t) \frac{d R_{\mathrm{CCA}}(t)}{d t}+R_{\mathrm{CCA}}(t)=R_{\mathrm{CCA}}^{*}(t) .
$$

This equation is at the basis of cold-wire anemometry in CCA operation. For the sake of clarity, the time dependence has been indicated in all the terms that are time-dependent.

From Fig. 2, the relation between the output voltage and the wire resistance $R_{\mathrm{CCA}}(t)$ is given by

$$
E_{\mathrm{CCA}}(t)=G\left\{100 I_{\mathrm{CCA}}\left[R_{\mathrm{CCA}}(t)+r_{L}\right]-E_{\text {offset }}\right\} .
$$

Note that no hardware correction of the time lag is provided by the electronic circuit. Therefore, a compensation procedure requires the differential equation Eq. (A8) to be solved during postprocessing. Equation (A8) is rewritten as Eq. (1) in the main text.

\section{APPENDIX B: BASIC CVA EQUATIONS FOR A HOT WIRE}

A simplified sketch of the CVA is given in Fig. 3. The main equations that describe a hot wire operated by a CVA are briefly presented in the following. The reader interested in more details should refer to the available literature, e.g., Sarma, ${ }^{16}$ Comte-Bellot, ${ }^{19,20}$ Comte-Bellot et al.,${ }^{21}$ or Berson et al. ${ }^{18}$ Note that although the time dependence is not explicitly mentioned in the following, one should keep in mind that $E_{\mathrm{CVA}}, R_{\mathrm{CVA}}$, and $I_{\mathrm{CVA}}$ are time-dependent. Only the voltage across the wire and its lead, $V_{w}=\left(R_{\mathrm{CVA}}+r_{L}\right) I_{\mathrm{CVA}}$, is kept constant by the feedback circuit.

The first basic equation relates the output voltage $E_{\mathrm{CVA}}$ to the actual resistance of the wire $R_{\mathrm{CVA}}$. This equation derives from Ohm's and Kirchhoff's laws, applied to the CVA circuit presented in Fig. 3. ${ }^{20,21}$ After some algebra one obtains

$$
E_{\mathrm{CVA}}=V_{w}\left[1+\frac{R_{2}}{R_{F}}+\frac{R_{2}}{R_{\mathrm{CVA}}+r_{L}}+T_{C} R_{2} \frac{d}{d t}\left(\frac{1}{R_{\mathrm{CVA}}+r_{L}}\right)\right] .
$$

$V_{w}$ is the constant voltage across the wire, adjustable up to 1 $\mathrm{V}$. The time factor $T_{C}=R_{2 a} R_{2 b} C / R_{2}$ creates a phase lead. It is a partial hardware correction of the thermal lag of the wire. In the experiments presented in Sec. IV, $T_{C}=100 \mu \mathrm{s}$. Other useful values are: $R_{2 a} \simeq R_{2 b} \simeq 50 \Omega, R_{2}=R_{2 a}+R_{2 b} \simeq 100 \Omega$, $R_{1}=1500 \Omega, R_{F}=400 \Omega$, and $R_{d} \gg R_{2}{ }^{16}$

The second basic equation relates the resistance of an ideal wire $R_{\text {CVA }}^{*}$ without thermal lag to the resistance of the actual wire $R_{\mathrm{CVA}}$ with thermal lag. This basic equation is deduced from the following heat balances for the ideal wire,

$$
0=V_{w}^{2} \frac{R_{\mathrm{CVA}}^{*}}{\left(R_{\mathrm{CVA}}^{*}+r_{L}\right)^{2}}-\left(R_{\mathrm{CVA}}^{*}-R_{a}\right) f[U(t)],
$$

and for the actual wire,

$$
\frac{m_{w} c_{w}}{\chi R_{0}} \frac{d R_{\mathrm{CVA}}}{d t}=V_{w}^{2} \frac{R_{\mathrm{CVA}}}{\left(R_{\mathrm{CVA}}+r_{L}\right)^{2}}-\left(R_{\mathrm{CVA}}-R_{a}\right) f[U(t)] .
$$

Eliminating $f[U(t)]$ in these two equations gives the following governing equation: 


$$
\begin{aligned}
\frac{1}{V_{w}^{2}} \frac{m_{w} c_{w}}{\chi R_{0}} \frac{d R_{\mathrm{CVA}}}{d t}= & \frac{R_{\mathrm{CVA}}}{\left(R_{\mathrm{CVA}}+r_{L}\right)^{2}} \\
& -\frac{R_{\mathrm{CVA}}^{*}}{\left(R_{\mathrm{CVA}}^{*}+r_{L}\right)^{2}} \frac{R_{\mathrm{CVA}}-R_{a}}{R_{\mathrm{CVA}}^{*}-R_{a}} .
\end{aligned}
$$

Up to now, no linearization was performed. Equation (B4) is a nonlinear differential equation that is used throughout the procedure to process CVA output data. Note that the coefficient $m_{w} c_{w} / \chi R_{0}$ appears in Eq. (B4). The same coefficient is also involved in the definition of the time lag of the CCA [see Eqs. (3) and (A7)]. This coefficient is intrinsic to the wire. A major advantage of the CVA is that $m_{w} c_{w} / \chi R_{0}$ can be determined separately, in a low-turbulence flow such as the potential core of a jet, in which Eq. (B4) can be linearized.

Consider small resistance fluctuations around a mean value, i.e., all the resistances are of the form:

$$
\begin{aligned}
& R_{\mathrm{CVA}}=\bar{R}_{\mathrm{CVA}}+r_{\mathrm{CVA}}^{\prime} \text { with } r_{\mathrm{CVA}}^{\prime} \ll \bar{R}_{\mathrm{CVA}} ; \\
& R_{\mathrm{CVA}}^{*}=\bar{R}_{\mathrm{CVA}}^{*}+r_{\mathrm{CVA}}^{\prime *} \text { with } r_{\mathrm{CVA}}^{\prime *} \ll \bar{R}_{\mathrm{CVA}}^{*} ; \\
& R_{a}=\bar{R}_{a}+r_{a}^{\prime} \text { with } r_{a}^{\prime} \ll \bar{R}_{a} .
\end{aligned}
$$

Substituting into Eq. (B4) and keeping only the first-order terms in the development leads to

$$
\bar{M}_{\mathrm{CVA}} \frac{d r_{\mathrm{CVA}}^{\prime}}{d t}+r_{\mathrm{CVA}}^{\prime}=r_{\mathrm{CVA}}^{\prime *} \text {. }
$$

Equation (B5) is a classical first-order differential equation characterized by the time constant $\bar{M}_{\mathrm{CVA}}$. The value of $\bar{M}_{\mathrm{CVA}}$ appears naturally during the linearization process and is given by

$$
\bar{M}_{\mathrm{CVA}}=\frac{m_{w} c_{w}}{\chi R_{0}} \frac{1}{V_{w}^{2}}\left(\bar{R}_{\mathrm{CVA}}+r_{L}\right)^{2} \frac{\bar{a}_{w}}{1+2 \bar{a}_{w}} L M .
$$

$L M$ is a factor that takes into account the resistance of the lead connecting the wire to the anemometer, ${ }^{21}$

$$
L M=\left[1+\frac{r_{L}}{R_{a}\left(1+\bar{a}_{w}\right)}\right]\left[1+\frac{r_{L}}{R_{a}\left(1+\bar{a}_{w}\right)\left(1+2 \bar{a}_{w}\right)}\right]^{-1} .
$$

$\bar{M}_{\mathrm{CVA}}$ is easy to measure with the CVA in a low-turbulence flow. Therefore, the coefficient $m_{w} c_{w} / \chi R_{0}$ is directly given by

$$
\frac{m_{w} c_{w}}{\chi R_{0}}=\bar{M}_{\mathrm{CVA}} V_{w}^{2} \frac{1}{\left(\bar{R}_{\mathrm{CVA}}+r_{L}\right)^{2}} \frac{1+2 \bar{a}_{w}}{\bar{a}_{w}} \frac{1}{L M} .
$$

Once the coefficient $m_{w} c_{w} / \chi R_{0}$ is known, the nonlinear Eq. (B4) is solved in order to obtain $R_{\text {CVA }}^{*}$. Then $f[U(t)]$ is deduced from Eq. (B2)

$$
f[U(t)]=\frac{V_{w}^{2}}{\left(R_{\mathrm{CVA}}^{*}+r_{L}\right)^{2}} \frac{R_{\mathrm{CVA}}^{*}}{R_{\mathrm{CVA}}^{*}-R_{a}} .
$$

In summary, the CVA output is postprocessed by applying successively Eqs. (B1), (B4), and (B9) in order to obtain $f[U(t)]$. The postprocessing of CVA data does not involve any linearization. The only linearization that appears in the present work occurs during the measurement of the coefficient $m_{w} c_{w} / \chi R_{0}$, which is performed independently in a lowturbulence flow.

${ }^{1}$ T. T. Yeh and C. W. Van Atta, J. Fluid Mech. 58, 233 (1973)

${ }^{2}$ L. Mydlarski and Z. Warhaft, J. Fluid Mech. 358, 135 (1998).

${ }^{3}$ R. A. Antonia, L. W. B. Browne, and A. J. Chambers, Rev. Sci. Instrum. 52, 1382 (1981).

${ }^{4}$ J. Lemay and A. Benaïssa, Exp. Fluids 31, 347 (2001).

${ }^{5}$ J. Lemay, A. Benaïssa, and R. A. Antonia, Exp. Therm. Fluid Sci. 27, 133 (2003).

${ }^{6}$ J. C. LaRue, T. Deaton, and C. H. Gibson, Rev. Sci. Instrum. 46, 757 (1975).

${ }^{7}$ K. Bremhorst and D. B. Gilmore, Int. J. Heat Mass Transfer 21, 145 (1978).

${ }^{8}$ K. Bremhorst and L. J. W. Graham, Meas. Sci. Technol. 1, 425 (1990).

${ }^{9}$ L. J. W. Graham and K. Bremhorst, Meas. Sci. Technol. 2, 238 (1991).

${ }^{10}$ P. V. Vukoslavčević and J. M. Wallace, Meas. Sci. Technol. 13, 1615 (2002).

${ }^{11}$ T. J. Pedley, J. Fluid Mech. 67, 209 (1975)

${ }^{12}$ D. F. Elger and R. L. Adams, J. Phys. E 22, 166 (1989).

${ }^{13}$ G. Huelsz and E. Ramos, J. Acoust. Soc. Am. 103, 1532 (1998).

${ }^{14}$ A. Berson, "Vers la miniaturisation des réfrigérateurs thermoacoustiques: Caractérisation du transport non-linéaire de chaleur et des écoulements secondaires," Ph.D. dissertation, Ecole Centrale de Lyon, 2007 (in French).

${ }^{15} \mathrm{Ph}$. Blanc-Benon and A. Berson, AIP Conf. Proc. 1022, 359 (2008).

${ }^{16}$ G. R. Sarma, Rev. Sci. Instrum. 69, 2385 (1998).

${ }^{17}$ G. R. Sarma and R. W. Lankes, Rev. Sci. Instrum. 70, 2384 (1999).

${ }^{18}$ A. Berson, Ph. Blanc-Benon, and G. Comte-Bellot, Rev. Sci. Instrum. 80, 045102 (2009).

${ }^{19}$ G. Comte-Bellot, in Handbook of Fluid Dynamics, edited by R. W. Johnson (CRC, Boca Raton, 1998), Chap. 34, pp. 1-29.

${ }^{20}$ G. Comte-Bellot, in Handbook of Experimental Fluid Mechanics, edited by C. Tropea, A. L. Yarin, and J. F. Foss (Springer, Berlin, 2007), Secs. 5.2.1-5.2.7, pp. 229-283.

${ }^{21}$ G. Comte-Bellot, J. Weiss, and J. C. Béra, Rev. Sci. Instrum. 75, 2075 (2004). 